\title{
Ein Fortpflanzungsmedizingesetz für Deutschland
}

\author{
Henning M. Beier • Martin Bujard · Klaus Diedrich • Horst Dreier • \\ Helmut Frister - Heribert Kentenich - Hartmut Kreß • \\ Jan-Steffen Krüssel • Annika K. Ludwig • Eva Schumann • \\ Thomas Strowitzki · Jochen Taupitz $\cdot$ Christian J. Thaler · Petra Thorn • \\ Claudia Wiesemann · Hans-Peter Zenner
}

Online publiziert: 31. Januar 2018

(C) Nationale Akademie der Wissenschaften Leopoldina 2018

\begin{abstract}
Die Erstveröffentlichung des Beitrags „Ein Fortpflanzungsmedizingesetz für Deutschland“ (Diskussion Nr. 13, Halle (Saale)) wurde herausgegeben durch die Nationale Akademie der Wissenschaften Leopoldina 2017 unter der ISBN 978-3-8047-3791-4.

Mit freundlicher Genehmigung der (C) Nationale Akademie der Wissenschaften Leopoldina 2017. All rights reserved.

Publikationen in der Reihe „Leopoldina Diskussion“ sind Beiträge der genannten Autorinnen und Autoren. Mit den Diskussionspapieren bietet die Akademie Wissenschaftlerinnen und Wissenschaftlern die Möglichkeit, Denkanstöße zu geben oder Diskurse anzuregen und hierfür auch Empfehlungen zu formulieren.
\end{abstract}

Prof. Dr. Dr. H. M. Beier

Institut für Molekulare und Zelluläre Anatomie, Universitätsklinikum RWTH Aachen, Aachen, Deutschland

Dr. M. Bujard

Bereich „Familie und Fertilität“, Bundesinstitut für Bevölkerungsforschung (BiB), Wiesbaden, Deutschland

Prof. Dr. K. Diedrich

Klinik für Frauenheilkunde und Geburtshilfe, Universitätsklinikum Schleswig-Holstein, Lübeck, Deutschland

Prof. Dr. H. Dreier

Lehrstuhl für Rechtsphilosophie, Staats- und Verwaltungsrecht, Julius-Maximilians-Universität Würzburg, Würzburg, Deutschland

Prof. Dr. H. Frister

Lehrstuhl für Strafrecht und Strafprozessrecht, Heinrich-Heine-Universität Düsseldorf, Düsseldorf, Deutschland

Prof. Dr. H. Kentenich

Fertility Center Berlin, Berlin, Deutschland

Prof. Dr. H. Kreß

Evangelisch-Theologische Fakultät, Abteilung Sozialethik, Rheinische

Friedrich-Wilhelms-Universität Bonn, Bonn, Deutschland 
Zusammenfassung Die rechtliche Regelung der Fortpflanzungsmedizin ist dringend reformbedürftig. Das Embryonenschutzgesetz von 1990 erfasst die neuesten technischen Entwicklungen nicht, ist in manchen Bereichen unstimmig und lückenhaft, setzt die betroffenen Frauen, Paare und Kinder unnötigen gesundheitlichen Risiken aus, erschwert paradoxerweise die Durchsetzung von Kinderrechten und erzeugt Gerechtigkeitsprobleme und Rechtsunsicherheit für die betroffenen Paare und die behandelnden Ärztinnen und Ärzte.

Das Embryonenschutzgesetz enthält zudem nur strafrechtliche Verbote. Diese erlauben keine angemessene Reaktion auf die medizinische Entwicklung und den gesellschaftlichen Wandel und werden der Komplexität der Materie nicht gerecht.

Diese Probleme müssen gelöst werden. Der Bundesgesetzgeber verfügt seit mehr als 20 Jahren über die Kompetenz zur Regelung der Fortpflanzungsmedizin. Er sollte in der kommenden Legislaturperiode ein umfassendes Fortpflanzungsmedizingesetz schaffen.

Prof. Dr. J.-S. Krüssel

Universitäres interdisziplinäres Kinderwunschzentrum Düsseldorf (UniKiD), Düsseldorf,

Deutschland

Prof. Dr. A. K. Ludwig

Praxis für Frauengesundheit und Pränatalmedizin, Hamburg, Deutschland

Prof. Dr. E. Schumann

Zentrum für Medizinrecht, Institut für Rechtsgeschichte, Rechtsphilosophie und Rechtsvergleichung,

Georg-August-Universität Göttingen, Göttingen, Deutschland

Prof. Dr. T. Strowitzki

Abt. Gynäkologische Endokrinologie und Fertilitätsstörungen, Universitätsklinikum Heidelberg, Heidelberg, Deutschland

Prof. Dr. J. Taupitz

Institut für Deutsches, Europäisches und Internationales Medizinrecht, Gesundheitsrecht und Bioethik der Universitäten Heidelberg und Mannheim, Mannheim, Deutschland

Prof. Dr. C. J. Thaler

Hormon- und Kinderwunschzentrum, Klinikum der Ludwig Maximilians Universität München,

München, Deutschland

Dr. P. Thorn

Praxis für Paar- und Familientherapie/Psychosoziale Kinderwunschberatung, Mörfelden,

Deutschland

Prof. Dr. C. Wiesemann $(\bowtie)$

Institut für Ethik und Geschichte der Medizin, Universitätsmedizin Göttingen,

Humboldtallee 36, 37073 Göttingen, Deutschland

E-Mail: cwiesem@gwdg.de

Prof. Dr. H.-P. Zenner

Ethik-Kommission an der Medizinischen Fakultät der Eberhard-Karls-Universität und am

Universitätsklinikum Tübingen, Universität Tübingen, Tübingen, Deutschland 


\section{Ein Fortpflanzungsmedizingesetz für Deutschland}

Die Fortpflanzungsmedizin ist in einer rasanten Entwicklung begriffen. Immer mehr Personen gründen mithilfe von Techniken der Fortpflanzungsmedizin eine Familie. Pro Jahr werden in Deutschland über 80.000 In-vitro Fertilisationen (,künstliche Befruchtungen“) durchgeführt. Weil aus gesellschaftlichen Gründen das Durchschnittsalter der Erstgebärenden steigt, aber die biologische Fruchtbarkeit im Alter abnimmt, wird die Zahl der Hilfesuchenden aller Voraussicht nach weiter ansteigen. Zudem werden weltweit Verfahren wie Eizellspende, Embryospende oder Leihmutterschaft zunehmend in Anspruch genommen. Da diese Verfahren wie andere Möglichkeiten der modernen Fortpflanzungsmedizin gewichtige ethische Fragen aufwerfen und wesentliche individuelle Rechte betreffen, bedarf es einer angemessenen rechtlichen Regelung

Allerdings ist die Rechtslage in Deutschland seit langem unzureichend. Das Embryonenschutzgesetz stammt von 1990 und ist damit fast 30 Jahre alt. Es erfasst viele neue reproduktionsmedizinische Entwicklungen nicht, was die Fortpflanzungsmediziner nicht selten $\mathrm{zu}$ einer dem heutigen internationalen Stand nicht mehr angemessenen Behandlung zwingt und zu unnötigen Risiken für Mutter und Kind führt. Darüber hinaus wird das Embryonenschutzgesetz auch dem gesellschaftlichen Wandel und der Vielfalt heutiger Familienformen nicht mehr gerecht. In Folge einander widersprechender gesetzlicher Regelungen im Embryonenschutzgesetz einerseits und im Familien- und Sozialrecht sowie ärztlichen Berufsrecht andererseits kommt es zur Ungleichbehandlung von verheirateten und unverheirateten, heterosexuellen und gleichgeschlechtlichen Paaren sowie alleinstehenden Personen. Die defizitäre Rechtslage führt oft auch zu Nachteilen für das Kind, weil die rechtliche ElternKind-Zuordnung unzureichend geregelt ist und viele Kinder ihr Recht auf Kenntnis der Abstammung nicht durchsetzen können. Viele weitere wichtige Fragen wie zum Beispiel die psychosoziale Beratung sind bislang ebenfalls gesetzlich nicht hinreichend geregelt. All dies zeigt, wie notwendig eine neue, umfassende Regelung der Fortpflanzungsmedizin ist. Zwar hat es in der Vergangenheit punktuelle Ergänzungen des geltenden Rechts gegeben, beispielsweise zur eingeschränkten Zulassung der Präimplantationsdiagnostik (PID) oder zur Errichtung eines Samenspenderegisters. Diese reichen jedoch bei weitem nicht aus.

Als besonders regelungsbedürftig erweisen sich folgende Punkte:

- In den fast drei Jahrzehnten seit der Verabschiedung des Embryonenschutzgesetzes haben sich auf dem Gebiet der Embryologie wesentliche neue wissenschaftliche Erkenntnisse ergeben, die unbedingt berücksichtigt werden müssen. ${ }^{1}$ Die Definition des Embryos in $\S 8$ Embryonenschutzgesetz etwa stützt sich auf überholte Vorstellungen von den zellbiologischen und molekulargenetischen Abläufen der Befruchtung und frühen Embryonalentwicklung. Auch der Begriff der Totipotenz, der als Kriterium für den Lebensschutz des menschlichen Embryos dient, hat sich

\footnotetext{
1 Die Leopoldina hat sich in der Vergangenheit bereits einzelnen Aspekten des Themas gewidmet: siehe beispielsweise die Stellungnahme „Präimplantationsdiagnostik (PID) - Auswirkungen einer begrenzten Zulassung in Deutschland“ 2011 (http://www.leopoldina.org/uploads/tx_leopublication/201101_ natEmpf_PID-DE.pdf).
} 
auf Grund neuer Forschungsergebnisse als nicht sinnvoll anwendbar erwiesen. Die Unschärfen des bestehenden Gesetzestextes erzeugen in der Praxis große Rechtsunsicherheit.

- Die medizinische Praxis der In-vitro Fertilisation (IVF) in zahlreichen europäischen Staaten folgt dem allgemein anerkannten internationalen Stand des Wissens, wonach von mehreren Embryonen geplantermaßen nur derjenige mit der größten Entwicklungsfähigkeit übertragen wird. Dieser sogenannte elektive Single-Embryo-Transfer vermeidet risikobehaftete und gesundheitsgefährdende Mehrlingsschwangerschaften, ohne dabei die individuelle Chance auf eine Schwangerschaft nennenswert zu verringern. Dieses Vorgehen ist in Deutschland jedoch bei Strafe untersagt. Im Gegensatz zu anderen Ländern werden deswegen in Deutschland Mehrlingsschwangerschaften mit Frühgeburten in Kauf genommen, die erhebliche Gesundheitsrisiken insbesondere für die Kinder mit sich bringen.

- Die Spende von Samenzellen ist in Deutschland erlaubt, die Eizellspende verboten. Während also infertile Männer mit Hilfe einer Keimzellspende eine Familie gründen können, ist diese Option Frauen, die etwa in Folge einer Krebserkrankung keine eigenen Eizellen mehr bilden können, verwehrt. Diese Ungleichbehandlung der Geschlechter lässt sich schwerlich rechtfertigen. Beim Verbot der Eizellspende im Embryonenschutzgesetz hat man sich auf die vermeintlich schädlichen Auswirkungen einer „gespaltenen Mutterschaft“ berufen. Mittlerweile ist jedoch durch Forschungen im Ausland belegt, dass keine bedeutsamen Nachteile für die Entwicklung und das Wohlbefinden der Kinder und die Eltern-KindBeziehung entstehen. Auch die Risiken für die Eizellspenderin können durch verbesserte Stimulationstechniken reduziert werden. Die Kommerzialisierung ließe sich durch stringente Vorgaben wie etwa bei der Lebendorganspende eindämmen. Aufgrund der restriktiven deutschen Gesetzeslage sehen sich viele Paare gezwungen, eine Eizellspende im Ausland in Anspruch zu nehmen. Dort wird oft die anonyme Eizellspende praktiziert, wodurch dem Kind das verfassungsrechtlich verbriefte Recht auf Kenntnis seiner Abstammung versagt bleibt. Insofern beeinträchtigt das im Embryonenschutzgesetz verankerte Verbot der Eizellspende indirekt das Kindeswohl.

- Die Embryospende/Embryoadoption wird in Deutschland mehr und mehr praktiziert, ist im Embryonenschutzgesetz aber nicht normiert. Hier hat schon der Deutsche Ethikrat 2016 eine umfassende gesetzliche Regelung angemahnt. ${ }^{2}$

- Bei der Zeugung eines Kindes unter Verwendung einer Samenspende sind die familienrechtlichen Folgen bislang nur für Kinder, die in eine Ehe hineingeboren werden, angemessen geregelt. Hingegen ist die rechtliche Zuordnung eines Kindes zum nichtehelichen Lebensgefährten der Mutter keineswegs gesichert und zur verpartnerten Co-Mutter nur über den Umweg der Stiefkindadoption möglich. Aus Gründen der Rechtssicherheit und vor allem zum Wohle des Kindes sollte der Gesetzgeber daher in allen Fällen ,gespaltener Elternschaft“ durch eine Reform des Abstammungsrechts dafür Sorge tragen, dass die rechtliche Zuordnung

\footnotetext{
2 Deutscher Ethikrat, Embryospende, Embryoadoption und elterliche Verantwortung, Stellungnahme, 2016 (http://www.ethikrat.org/dateien/pdf/stellungnahme-embryospende-embryoadoption-und-elterlicheverantwortung.pdf).
} 
des Kindes zum Wunschelternteil verbindlich mit der Geburt bzw. zeitnah nach der Geburt erfolgt. Der vom Bundesministerium der Justiz und für Verbraucherschutz (BMJV) eingesetzte Arbeitskreis Abstammungsrecht hat erst vor kurzem (Juli 2017) Thesen vorgelegt ${ }^{3}$, die als Grundlage für eine Reform herangezogen werden könnten.

- Besonders schwierige ethische und rechtliche Fragen wirft die in Deutschland verbotene Leihmutterschaft auf. Unabhängig von der stark umstrittenen Frage einer rechtlichen Zulassung der Leihmutterschaft in der Zukunft besteht aber schon heute Regelungsbedarf für die im Ausland von einer Leihmutter geborenen und dann in Deutschland aufwachsenden Kinder. Auch in diesen Fällen ist die rechtliche Eltern-Kind-Zuordnung von elementarer Bedeutung für das Wohl dieser Kinder. Von ihr hängen zahlreiche Rechtsfolgen wie die elterliche Sorge, Unterhaltsansprüche und die Staatsangehörigkeit ab. Hierfür sind gesetzliche Lösungen erforderlich.

- Auch in Deutschland werden mittlerweile an vielen fortpflanzungsmedizinischen Zentren Eizellen eingefroren (kryokonserviert). Zum Teil geschieht dies aus medizinischen Gründen (z. B. vor einer chemotherapeutischen Behandlung), zum Teil aus sozialen Gründen (,social freezing“). Im Interesse der Frau, des Paares und des zukünftigen Kindes sollten die Rahmenbedingungen für die Aufbewahrung, Befruchtung und Übertragung von Eizellen geregelt werden.

- Das Embryonenschutzgesetz verbietet die Befruchtung einer Eizelle mit der Samenzelle eines Mannes nach dessen Tod. Die Reichweite dieses Verbots ist allerdings umstritten. Höchstgrenzen für die Aufbewahrung von Samenzellen, Eizellen bzw. Embryonen enthält das Embryonenschutzgesetz nicht. Auch hier besteht gesetzlicher Klärungsbedarf.

Während in unseren Nachbarländern Österreich und der Schweiz seit Jahrzehnten umfassende Fortpflanzungsmedizingesetze vorliegen, die zudem 2015 bzw. 2017 grundlegend reformiert wurden, ist die deutsche Rechtslage noch immer von dem 1990 als reines Strafgesetz konzipierten Embryonenschutzgesetz geprägt. Damals war ein Fortpflanzungsmedizingesetz auf Bundesebene wegen fehlender Zuständigkeit des Bundes gemäß Art. 74 GG nicht möglich. Allerdings wurde die Gesetzgebungskompetenz des Bundes 1994 bzw. 2006 auf die Fortpflanzungsmedizin erweitert. Er verfügt also über die Möglichkeit, ein Fortpflanzungsmedizingesetz zu erlassen, in dem neben strafrechtlichen Verbotsnormen die auf dem Gebiet der Biomedizin so wichtigen verwaltungsrechtlichen und -organisatorischen, aber ggf. auch die erforderlichen bürgerlich-rechtlichen (insbesondere familienrechtlichen) und sozialrechtlichen Regelungen in organischer Weise zusammengeführt werden. Nur in einem solchen umfassenden Fortpflanzungsmedizingesetz kann die komplexe Materie angemessen geregelt werden.

\footnotetext{
3 Bundesministerium der Justiz und für Verbraucherschutz (Hrsg.), Abschlussbericht des Arbeitskreises Abstammungsrecht - Empfehlungen für eine Reform des Abstammungsrechts, 2017 (http://www.bmjv.de/ SharedDocs/Downloads/DE/Artikel/07042017_AK_Abstimmung_Abschlussbericht.html).
} 


\section{Weitere Veröffentlichungen aus der Reihe „Leopoldina Diskussion““}

- Nr. 12: Antibiotika-Forschung: 5 Jahre danach. Was hat sich getan, was bleibt zu tun? - 2017

- Nr. 11: Nachhaltige Zeitenwende? Die Agenda 2030 als Herausforderung für Wissenschaft und Politik - Dokumentation des Leopoldina-Symposiums vom 18. Oktober 2016 in Berlin - 2017

- Nr. 10: Ethische und rechtliche Beurteilung des genome editing in der Forschung an humanen Zellen - 2017

- Nr. 9: Gutes Leben oder gute Gesellschaft? - 2017

- Nr. 8: Tiefe Hirnstimulation in der Psychiatrie - Zur Weiterentwicklung einer neuen Therapie - 2017

- Nr. 7: Zum Verhältnis von Medizin und Ökonomie im deutschen Gesundheitssystem -8 Thesen zur Weiterentwicklung zum Wohle der Patienten und der Gesellschaft -2016

- Nr. 6: Sprache der Wissenschaft - Sprache der Politikberatung. Vermittlungsprozesse zwischen Wissenschaft und Politik - 2015

- Nr. 5: Transplantationsmedizin und Organallokation in Deutschland: Probleme und Perspektiven - 2015

- Nr. 4: Freiheit und Verantwortung der Wissenschaft: Rechtfertigen die Erfolgschancen von Forschung ihre potentiellen Risiken? Dokumentation des Symposiums der Nationalen Akademie der Wissenschaften Leopoldina, der Deutschen Forschungsgemeinschaft und des Deutschen Ethikrates am 3. November 2014 in Halle (Saale) - 2015

- Nr. 3: Die Synthetische Biologie in der öffentlichen Meinungsbildung. Überlegungen im Kontext der wissenschaftsbasierten Beratung von Politik und Öffentlichkeit -2015

- Nr. 2: Auf dem Wege zur perfekten Rationalisierung der Fortpflanzung? Perspektiven der neuesten genetischen Diagnostik. Dokumentation des Leopoldina-Gesprächs am 16. und 17. Februar 2013 in Halle (Saale) - 2014

- Nr. 1: Die Zukunftsfähigkeit des deutschen Wissenschaftssystems. Für die nachhaltige Entwicklung von Forschung, Lehre und Wissenstransfer - 2013 